\title{
Spelling of pseudowords and real words in Dutch-speaking children with and without dyslexia
}

\begin{abstract}
The purpose of this study was to explore the spelling knowledge and skills of elementary school children with and without dyslexia who speak Dutch, a language with a relatively transparent orthography. Children in grades two to six completed real and pseudoword spelling dictation tasks. Spelling performance was compared in 218 children with $(n=55)$ and without $(n=163)$ dyslexia. There was a medium effect size for morphological, phonological, combined, and etymological spelling skills for differences between students with and without dyslexia. In addition, spelling real words resulted in medium effect sizes, whereas spelling pseudowords resulted in small effect sizes. Children without dyslexia performed above the mean for the entire sample in grade 4 , whereas same-age peers with dyslexia did so two years later. Even so, Dutch-speaking children with dyslexia continued to have difficulty spelling words requiring phonological skills and spelling pseudowords even up to grade 6. Clinicians are encouraged to be aware of the importance of the choice of spelling items in the assessment of dyslexia. The use of pseudowords appears to be helpful in older children to identify spelling problems.
\end{abstract}




\section{INTRODUCTION}

When children learn to spell, they learn to map sounds to letters. This mapping is not always regular or predictable, resulting in differences in the degree of sound-letter transparency in alphabetic languages. There is a continuum from languages with an opaque or nontransparent orthography, such as English, to languages with a shallow or transparent and consistent orthography, such as Italian and Spanish (Wimmer \& Landerl, 1997). Dutch is a language that is relatively transparent in phoneme-grapheme correspondence. Studies have revealed that learning to spell is faster in transparent orthographies than in opaque writing systems (Tops, Callens, Bijn, \& Brysbaert, 2014).

Across languages a large body of research provides support for reading proficiency (Jap, Borleffs, \& Maassen, 2017; Kahmi \& Hinton, 2000), vocabulary knowledge (Hilte \& Reitsma, 2011) and verbal working memory (De Weerdt, Desoete \& Roeyers, 2013; Jongejan, Verhoeven, \& Siegel, 2007) as general predictors of spelling ability in young children. However there is less consensus on the more specific and perhaps more language-dependent subskills that are involved across languages (Duranovic, 2017).

\section{Spelling Subskills in Dutch}

The spelling ability of Dutch elementary school childeren has been found to be influenced by the phonological, orthographic, and morphological subskills (Keuning \& Verhoeven, 2007). In addition, the spelling of words with ambiguous spelling patterns that arise from varied lingusitic origins has to be 'memorized' (Tops et al., 2014), and this represents etymological subskills. Automatized integration of these subskills frees cognitive resources for text comprehension and production (Coch, Mitra, \& George, 2012; Daigle, Costerg, Plisson, Ruberto, \& Varin, 2016). Beginning spellers in particular (Coch et al., 2012; Hilte \& Reitsma, 2006; Reitsma, 1983) in transparent languages (Tolchinsky, Liberman, \& Alonso-Cortés Fradejas, 2015) depend on phonological skills to translate phonemes into 
corresponding graphemes based on a more or less one-to-one phoneme-grapheme or soundspelling correspondence. In Dutch, a large number of monosyllabic words without (e.g., ' $k a t$ ' [cat]) and with (e.g., $k r$ in 'krat' [box]) consonant clusters and disyllabic words (e.g., 'kantoor' [office]) can be spelled based on phonological skills. In addition, phonological skills also have to be applied at the syllable level. For instance, the speller has to apply spelling rules (orthographic knowledge) based on the duration of the sound of the vowel at the end of the pronounced syllable. So, the sound context directs the transcription of the word. When the final syllable contains a short vowel, a double consonant is used to denote this (e.g., 'katten' [cats]). When a long vowel is heard in the final syllable, a single consonant is used to denote this (e.g., 'apen' [apes]).

With increased exposure to written words, orthographic skills become important (Hilte \& Reitsma, 2011; Mather \& Wending, 2012). For instance, children build up an implicit knowledge of how trigraphs (e.g., 'ooi') map to the corresponding sound ('oj') and of sequences and combinations of letters in their own language. In Dutch, these skills are involved in the spelling of words with complex phoneme-grapheme correspondences (e.g., sound 'aj'= of grapheme 'aai' as in 'maai' [mow]).

To determine the contribution of phonological and orthographic skills to spelling, there might be some merit in comparing the spelling of real and pseudowords (Campbell, 1985; Jongejan et al., 2007; Tellings \& Bouts, 2011; Van Vreckem \& Desoete, 2016). Pseudowords activate lexical and sublexical units in terms of shared letter sequences with real words. Pseudowords can only be spelled via mapping sounds to letters, whereas real words can also be spelled via direct recall of orthographic images due to a repeated co-occurrence of form and meaning (Hilte \& Reitsma, 2011). Real words and pseudowords are processed similarly by beginning first graders (Coch et al., 2012). In older children, research reveals a small effect of word length (Tellings \& Bouts, 2011) and existing words are spelled more 
accurately and more fluently compared to less familiar letter strings such as those occurring in pseudowords (Coch et al., 2012). Comparing spelling errors on real and pseudowords may help gain a better understanding of the nature of the subskills involved in spelling.

Research has shown that morphological skills also influence spelling (Daigle, Costerg, Plisson, Ruberto, \& Varin, 2016; Koh, Shakory, Chen, \& Deacon, 2017; Rispens, McBrideChang, \& Reitsma, 2008), enabling the recognition, manipulation, and use of the morphemic structure of words (Rispens et al., 2008; Tops et al., 2014). In Dutch, the final letter of the word 'mond' [mouth] is pronounced /t/ (i.e., it is devoiced) but written as ' $\mathrm{d}$ ' because the voiced / $\mathrm{d} /$ is audible in the plural form 'monden' [mouths]. This skill is also involved in lexical compounding--combining two free morphemes into a new word (e.g., 'slaapkamer' [bedroom])--and in inflectional and derivational morphology when new words are formed through the combination of a word stem and affixes (e.g., verlaat [leave]). Especially in less transparent languages, morphological information is needed to recover the correct spelling (Tolchinsky et al., 2015).

Finally, for words with unclear or arbitrary spelling deviations, the spellings must be memorized because phonological and morphological skills alone do not help and the spelling cannot be reconstructed on the basis of a rule (Tops et al., 2014). These words remain difficult to spell often because of the word's etymology (Keuning \& Verhoeven, 2007; Tops et al., 2014; Van Vreckem \& Desoete, 2016). Thus, complex loanwords (e.g., 'jury', 'chimpansee' [chimpanzee]) and ambiguous words that have the same sound but different graphemes (e.g., ei/ij sound the same in the words ' $k i j k$ ' [look], 'keizer' [emperor]) have to be stored in memory.

\section{Impaired Spelling in Dyslexia}

Dyslexia occurs in all languages (Joshi \& Carreker, 2009; Shaywitz, Morris, \& Shaywitz, 2008). However, the prevalence differs across countries (Callens, Tops, \& 
Brysbaert, 2012) with orthographic transparency having a major effect (Jap, Borleffs \& Maasen, 2017; Ziegler \& Goswami, 2005). According to the International Dyslexia Association, dyslexia refers to a neurodevelopmental disorder characterized by difficulties with accurate and/or fluent word recognition and by poor spelling and decoding abilities. In addition, Ghesquière (2014) proposed that skills had to be substantially below $\left(<10^{\text {th }}\right.$ percentile) those expected for the individual's chronological age and that reading problems must persist despite the provision of interventions that target specific reading difficulties. Thus, children with dyslexia have great difficulty learning to read and write, despite adequate learning opportunities (Bourassa \& Treiman, 2014). However, clear differences in rates of dyslexia are observed between transparent and nontransparent orthographies (Bergmann \& Wimmer, 2008). Duranovic (2017) notes there is a great deal of merit in understanding the nature of the spelling errors in different languages, as it reveals differences about the varying importance of those subskills with which children with dyslexia appear to struggle.

Although most researchers have agreed that spelling errors in dyslexia might at least partly be due to poor phonological skills (Angelelli et al., 2004; Caravolas \& Voli'n, 2001; Duranovic, 2017; Rello, Beaza-Yates, \& Llisterri, 2017; Tops et al., 2014), making the acquisition of the sound-to-letter mapping in transparent languages challenging (Tolchinsky et al., 2015), not all studies (e.g., Daigle, Costerg, Plisson, Ruberto, \& Varin, 2016) have revealed such deficiencies. Moreover, the body of research reveals age-related effects (Landerl \& Wimmer, 2000) in spelling errors within transparent languages. For instance, Landerl and Wimmer (2000) demonstrated that younger children with dyslexia in Germany (with a transparent orthography) made phonological errors up until grade 2, but older children made more orthographic-related spelling errors. In other transparent languages, such as Greek, the difficulties with phonological skills remain present in older children (Nikolopoulos, Goulandris, \& Snowling, 2003) compared to age-matched peers without 
dyslexia. In Dutch-speaking adolescents, spelling errors are classified as phonological or orthographic, with a slight dominance of phonological spelling errors (Tops et al., 2014), though there is also clinical evidence of children with dyslexia encountering difficulties because of morphological considerations (Koh et al., 2017) or because of a word's etymology (Van Vreckem \& Desoete, 2016).

\section{OBJECTIVES}

This study aims to investigate spelling skills in Dutch-speaking children with and without dyslexia to address the following research issues. First, the current study investigated if children with dyslexia in Flanders differed from peers without dyslexia on phonological, orthographic, morphological, and etymological skills. We wished to determine, for example, if children with dyslexia display difficulties on all these facets of spelling from grade 2 through 6 or if phonological errors appear with lower frequency among older children. As such, this study contributes to theory-building about spelling acquisition in children with and without a clinical diagnosis. Second, this study expands previous research in Dutch on spelling performance by analyzing the spelling of real words and pseudowords. The use of two- and three-syllable pseudowords expands previous studies in Dutch in which only monosyllabic pseudowords were employed (Campbell, 1985; Jongejan et al., 2007). In addition, testing pseudoword spelling is often done in clinical practice. We aim to investigate the usefulness of assessing pseudowords to differentiate children with and without dyslexia where the retrieval of meaning is less relevant to spelling performance. Finally, several studies suggest that subskills play a role in the acquisition of spelling, but it remains unclear what subskills are important and at what ages these subskills play a role in learning to spell even in a language with a relatively transparent orthography like Dutch. Therefore, the present study investigates the contribution of subskills and the relationships between the subskills to spell real and pseudowords in children with and without dyslexia. We wish to arrive at a more 
detailed categorization of spelling subskills and undertake an analysis of the kinds of knowledge associated with successful and less successful spelling performance.

\section{METHOD}

\section{Participants}

Each child was screened for inclusion in the study. Parents who had given their permission for their child to participate completed questions about previous diagnoses or other (medical) problems encountered by their child. Only native Dutch-speaking children from regular elementary schools in grade 2 through 6 with an average intelligence above 84 and without reported histories of visual or hearing impairment, brain damage, a chronic medical condition, insufficient instruction, serious emotional or behavioral disturbance, or developmental disorders (including behavior problems, autism, or motor disorders) were included. Prior to the study, reading and spelling skills were assessed to form two groups: children with dyslexia and children without learning problems.

To be included in the group of children with dyslexia, the child had to demonstrate a clinical diagnosis of dyslexia based on the International Dyslexia Association's criteria and on the three criteria used in Flanders (Ghesquière, 2014). There were 5 children ( 3 boys) from grade 2, 9 children ( 7 boys) from grade 3,17 children (13 boys) from grade 4,15 children (12 boys) from grade 5, and 9 children ( 5 boys) from grade 6 . All these children: (a) had substantial $\left(<10^{\text {th }}\right.$ percentile $)$ difficulties with reading abilities compared to those expected for the individual's chronological age and (b) their reading and spelling problems persisted despite the provision of interventions that target those difficulties (all children in this group received individual therapy for reading and spelling skills by a speech-language pathologist). Reading of real words was assessed using the One-Minute Reading Test (Brus \& Voeten, 1999), a standardized word reading test that requiered students to read as many words as possible in one minute from a list of words. The test-retest reliability varied from .82 till .92. 
(Prodiagnostiek, s.d.). Reading was also assessed using the Klepel Test (Van den Bos, Spelberg, Scheepstra, \& de Vries, 1994), a standarized pseudoword reading test that required students to read a many pseudo-words as possible in two minutes from a list of pseudowords. The reliability varied between .89 and .95 (Prodiagnostiek, s.d.) Both tests have good psychometric properties and are often used in other studies (e.g., De Weerdt et al., 2013; Rispens et al., 2008) and both have a test mean of 10 and standard deviation of 3. Spelling was assessed with the Paedological Institute Dictation Test (PI-Dictation; Geelhoed \& Reitsma, 2000). Finally, these children came from working- and middle-class socio-economic backgrounds and were Caucasian monolingual Dutch-speaking children. The group with dyslexia had a mean standard score of $3.43(\mathrm{SD}=2.21)$ and $5.28(\mathrm{SD}=1.72)$ on the OneMinute Reading Test and Klepel Test, respectively. The group with dyslexia also had a mean percentile of $3.70(\mathrm{SD}=7.87)$ on the PI-Dictation. In addition, their mean Full Scale IQ assessed with the Dutch version of the WISC-III (Kort et al., 2005; Wechsler et al., 2005) was $99.74(\mathrm{SD}=9.79)$. The mean Verbal IQ was $100.13(\mathrm{SD}=10.31)$ and the mean Performance IQ was $99.46(\mathrm{SD}=11.34)$.

The group of children without learning problems included 13 children (9 boys) from grade 2, 27 children ( 21 boys) from grade 3, 51 children (39 boys) from grade 4, 45 children (36 boys) from grade 5, and 27 children ( 15 boys) from grade 6 . All these children had at least average scores on standardized tests of reading and spelling used in schools. The children included in this group had a mean percentile of $46.28(S D=28.68)$ for reading and $49.44(S D$ $=26.43)$ for spelling. In addition classroom teachers rated their reading $(M=5.82 ; \mathrm{SD}=$ $0.94)$, spelling $(M=5.47 ; \mathrm{SD}=1.21)$, and intelligence $(M=5.58 ; \mathrm{SD}=1.43)$ as at least above average on a 7-point Likert scale.

The final sample consisted of 163 typically developing children, and 55 children with dyslexia. Mean age was 10 years, 3 months for children in the dyslexia group and 10 years, 4 
months for children in the group without learning problems. The schools from which both groups were recruited had similar social and educational characteristics insofar as parents' backgrounds were comparable, the time devoted to spelling instruction was similar (spelling was explicitly taught in all schools), and the reading and spelling curriculum content was similar. Teachers also had equivalent backgrounds: in Flanders, teachers on average have 16.72 years $(\mathrm{SD}=9.93)$ of experience in education (Van Steenbrugge, Valcke, \& Desoete, 2010).

\section{Instrument}

Real words and pseudowords were selected from the Spelling Test, ST1-6 (Van Vreckem \& Desoete, 2016), a norm-referenced spelling test based on the Dutch/Belgian spelling curriculum, for the present study. The predictive validity of the ST1-6 (Van Vreckem \& Desoete, 2016) has been demonstrated with a sample of 3,664 children. There was a significant correlation of .63, .66, .71, .80, and .70 between the ST 1-6 and the Dutch standardized spelling test used in all schools in grades 2, 3, 4, 5, and 6, respectively, and a significant correlation of between .53 (in grade 2) and .70 (in grades 3 and 5) between the ST1-6 and the exam results for spelling of the children.

All items were tested in a pilot study (data presented below) in order to determine their usefulness for this age group and their usedfulness in measuring individual differences in phonological, orthographic, morphological, and etymological skills in children with and without dyslexia. The 125 real words were dictated within a sentence; only the target word had to be written down. Overall, there was a mix of 51.59\% frequently occurring real words (e.g., oor [ear], vliegveld [airport], schoolreis [school trip]) and $48.41 \%$ infrequently occurring real words (e.g., bok [goat], snoei [prune], bekendste [most famous], bloemkool [cauliflower]). The 29 pseudowords (e.g., it, krinnen, kleeuw, onkprang) were presented after children completed spelling real words within the dictated sentences. Overall Cronbach's alpha for real words was .98 and for pseudowords was .81 . 
Because of the transparency of Dutch orthography, there was an oversampling of items that had to be spelled based on phonological skills in the ST1-6. In addition, the sample of words and pseudowords was guided by instruction in Belgium. Children learn to spell monosyllabic words without consonant clusters (e.g., put [pit]) in grade 1, monosyllabic words with consonant clusters (e.g., post [mail]) and disyllabic words (e.g., kuiken [chick]) in grade 2, and multisyllabic words (e.g., fluisteren [to whisper]) in grade 3, while they learn to spell words reliant on phonological skills at the syllable level that also require the application of a spelling rule from grades 2 through 4 (e.g., toverbol [magic wand], pianootje [small piano]). There were 32 real monosyllabic words depending on phonological skills (e.g., oor [ear], put [pit], koorts [fever], spring [jump], spoelt [rinses]) with Cronbach's alpha of .64 and scores with $M=30.89, S D=1.61$, and 7 real multisyllabic words (e.g., kuiken [chick], drempel [threshold], poorten [gates], mengen [mixing], karton [cartboard], fluisteren [whispering], kletsnat [very wet]) depending on the same phonological skills (Cronbach's alpha $=.81 ; M=5.81, S D=1.71)$ for the pilot study. There were also 15 real words depending on phonological skills at the syllable level requiring the application of a spelling rule (e.g., na [after], boten [boats], zussen [sisters], overvallers [robbers]) with Cronbach's alpha of .93 (M $=9.37, S D=4.66)$. In addition, 18 pseudowords depended on phonological skills (e.g., it, oos, buut, berfst, stor, truins; Cronbach's alpha $=.63 ; M=13.52 ; S D=2.63$ ) and 8 pseudowords depended on this skill at the syllable level (e.g., krinnen, peven, zumak, tronnen; Cronbach's alpha $=.80 ; M=4.10 ; S D=2.53)$. There were 9 items included to assess orthographic skills (Cronbach's alpha $=.50 ; M=7.46, S D=1.29)$, namely 6 real words (kraai [crow], schroef [screw], enkeling [individual]; $M=5.48 ; S D=0.77$ ) and 3 pseudowords (e.g., kleeuw, onkprang; $M=1.98 ; S D=0.83$ ) in the pilot study.

There also were 16 words that required morphological skills such as dealing with final 't' and 'p' (e.g., writing 'd' in mond [mouth]) and derivations and compound words (e.g., 
gewoon [ordinary], verhaal [story], handdoek [towel]; Cronbach's alpha =.87, $M=11.28 ; S D$ $=4.03$ ) and 28 words that depended on etymological skills (e.g., kijk [look] with 'ij' and not 'ei', blauwe [blue] with 'au' and not 'ou', verkouden [caught a cold] with 'ou' and not 'au', nicht [niece] with 'cht' to be remembered, acteur [actor]; Cronbach's alpha $=.93 ; M=14.58$; $S D=6.79)$. Finally, 21 words were selected that could only be written based on a combination of skills (e.g., allerlei [all kinds of], nijlpaard [hippopotamus], magisch [magic], auto-ongeluk [car accident], Albanië [Albania]; Cronbach's alpha $=.93 ; M=7.65 ; S D=6.04$ ) in the pilot study.

\section{Procedure}

To guarantee a standardized administration for all children, the ST1-6 was administrated in January or June according to the instructions in the manual. For the study reported here, all raw scores (number of correct items for each set of words) were subsequently converted to z-scores. All z-scores were based on the entire sample. All responses were scored for accuracy by three independent raters blinded to the research purposes. The interrater reliability was high at .99 .

\section{RESULTS}

To address our first research issue, phonological, orthographic, morphological, and etymological skills of Dutch-speaking children with dyslexia were compared to age-matched peers without dyslexia. For phonological and orthographic skills, the results from spelling accuracy with real words and pseudowords were investigated together. Because assumptions of normality were not met, non-parametric tests (Mann-Whitney U) were performed to evaluate the differences in the z-scores and the magnitude of differences as effect sizes $(\mathrm{r}=$ $\mathrm{Z} / \sqrt{ } \mathrm{N})$ were calculated.

The Mann-Whitney analyses (with effect-sizes) revealed significant differences between children with and without dyslexia on phonological skills when spelling 
monosyllabic words $(U=2394.00, Z=-5.51, p<.001, \mathrm{r}=-0.37)$, multisyllabic words $(U=$ 3292.00, $Z=-3.24, p=.002, \mathrm{r}=-0.22)$, and words for which spelling rules must be employed at the syllable level $(U=3232.50, Z=-3.12, p=.002, \mathrm{r}=-0.21)$. Children without dyslexia had above average skills from grade 4 on, whereas these items remained relatively more difficult for children with dyslexia. The magnitude of the differences between children with and without dyslexia on these different words was largest for real words $(U=1918.00, Z=-$ $6.38, p=.000, \mathrm{r}=-0.43)$. Overall, there was a significant difference between children with and without dyslexia with these subskills, and the largest difference was when phonological skills were recruited for spelling real words. For an overview see Table 1.

There were also significant differences between children with and without dyslexia when spelling words based on orthographic $(U=3727.50, Z=-2.17, p=.030, \mathrm{r}=-0.15)$, morphological $(U=1875.50, Z=-6.49, p<.001, \mathrm{r}=-0.41)$, and etymological $(U=2573.50, Z$ $=-4.73, p<.001, \mathrm{r}=-0.32$ ) patterns. Children with dyslexia had more problems with derivations, final ' $t$ ', and compounds compared to their age-matched peers without learning problems. They also had more problems with the combination of spelling skills $(U=2319.00$, $Z=-5.36, p<.001, \mathrm{r}=-0.36)$. Spelling real words dependent on orthographic skills was easier for all children from grade 4 on, and spelling pseudowords dependent on orthographic skills was relatively harder for children with dyslexia in grade 4, but not in grades 5 or 6 . Items depending on morphological, etymological, and combined skills could be spelled relatively accurately in grade 6 by children with dyslexia, whereas their matched peers without dyslexia were relatively successful with these words from grade 4 onward.

To address the second research issue, the spelling of real words and pseudowords was compared in children with and without dyslexia. In general on the total test pseudoword spelling $(U=3178.00, Z=-3.23, p=.001, \mathrm{r}=-0.22)$ was less informative (lower effectsize ' $r$ ') than real word spelling $(U=2051.00, Z=-5.98, p<.001, \mathrm{r}=-0.41)$ for differentiating 
Dutch-speaking children with and without dyslexia on their skills. However, children without learning problems (see Table 1) had above average spelling scores from grade 4 onward, whereas their peers with dyslexia had results above the mean on just real words two years later, in grade 6 and they did not score above the mean on pseudowords in grade 6 , pointing to their difficulties with the spelling of these kind of items where there is no repeated cooccurrence of form and meaning.

When differentiating phonological from orthographic skills, the spelling of real words based on phonology $(U=1918.00, Z=-6.38, p=.000, \mathrm{r}=-0.43)$ was more informative (higher effectsize ' $r$ ') compared to the spelling of phonological pseudowords ( $U=3210.00, Z$ $=-3.16, p=.002, \mathrm{r}=-0.21)$. For orthographic items the spelling of real words $(U=3725.50$, $Z=-2.17, p=.030, r=-0.15$ ) was as informative (same effectsize ' $r$ ') as the spelling of orthographic pseudowords $(U=3663.00, Z=-2.17, p=.030, \mathrm{r}=-0.15)$.

To address the last research issue, the correlations were computed between the accuracy scores on different kinds of words for children with dyslexia and their age-matched peers without dyslexia (see Table 2). For each group, all scores for various types of words correlated significantly, though the magnitude of correlations were generally higher for children with dyslexia.

\section{DISCUSSION}

The spelling ability of elementary school childeren has been found to be influenced by phonological, orthographic, and morphological subskills (Keuning \& Verhoeven, 2007). Different types of spelling errors have been documented in children with and without dyslexia even within transparent languages (Angelelli et al., 2004; Caravolas \& Voli’n, 2001; Daigle et al., 2016; Duranovic, 2017; Landerl \& Wimmer, 2000, Masterson \& Apel, 2000, 2010; Tops et al., 2014), suggesting the kinds of knowledge and skills recruited for spelling are diverse. This study investigated phonological, morphological, orthographic and 
etymological spelling skills in children with and without dyslexia in a relatively transparent language. In addition, it expanded previous findings in the Dutch language by focusing on existing real words and pseudowords.

First, in line with Daigle et al. (2016), Kahmi and Hinton (2000), Rello et al. (2017), and Tops et al. (2014), this study confirmed that children with dyslexia made more spelling errors than children without dyslexia. In general, these children were two years behind in their spelling accuracy compared to age-matched peers. In addition, and in agreement with findings reported by Angelelli et al. (2004), Caravolas and Voli'n, (2001), Daigle et al. (2016), and Duranovic (2017), phonological skills differentiated individuals with and without dyslexia in Dutch spoken in Flanders with a medium effect size. There was a significant difference between Dutch-speaking children with and without dyslexia on all phonological skills, with the largest effect size for the spelling of real monosyllabic words. It seems that Dutchspeaking children with dyslexia already experience serious problems with simple monosyllabic words, although this is the simplest spelling pattern in which there is mapping of individual sounds (phonemes) to letters (graphemes). This finding suggests poor phonemic awareness skills as those reported by Masterson and Apel (2010) in English and by Duranovic (2017) in the Bosnian language, which also has a transparent orthography (phonological errors were dominant in children with dyslexia at all grade levels in that study). However, in our study other errors were present, as has been reported with Dutch-speaking adolescents (Tops et al., 2014).

Second, our results with Dutch-speaking children are aligned with those reported by Kahmi and Hinton (2000), Nikolopoulos and colleagues (2003), Koh and colleagues (2017), and Tops et al. (2014) regarding the importance of morphological skills even in transparent languages. The children with dyslexia in our sample only performed above the mean for the sample on these words in grade 6 whereas their peers without learning problems did so from 
grade 4 onward. Previous studies have revealed poor morphological skills in children with reading problems and dyslexia throughout elementary school (Daigle et al., 2016; Koh et al., 2017; Landerl \& Wimmer, 2000; Masterson \& Apel, 2010). In Dutch-speaking children, words requiring recruitment of morphological skills to accurately spell differentiated children with and without dyslexia to the greatest degree (with an effect size of -.41).

Third, there was a small effect size for the differences between Dutch-speaking children with and without dyslexia for orthographic skills. These results have to be interpreted carefully due to the low internal consistency of this portion of the ST1-6.

Fourth, there was a medium effect size for the differences between children with and without dyslexia for etymological spelling skills. It appears Dutch-speaking children with dyslexia seem to lack the skills needed to memorize words with unclear or arbitrary spelling deviations and inconsistency in phoneme-grapheme correspondences (e.g., kijk [look] versus 'schoolreis' [school trip] with 'ij'/‘ei' and blauwe [blue] versus 'verkouden '[caught a cold] with 'au'/'ou'). Children with dyslexia could spell words relatively accurately based on etymology, according to our categorical definition, in grade 6 , whereas their peers without learning problems performed as well two years earlier (in grade 4) when spelling such words. Some might describe success in spelling words of this kind as orthographic awareness.

Finally children with dyslexia differed from their peers with a medium effect size in words that required a combination of spelling skills. They performed adequately (compared to the entire sample) on this task by grade 6 , whereas their peers without learning problems did so by grade.

To conclude, in line with recent research in children with a Bosnian (Duranonci, 2017) or French (Koh et al., 2017) language across grades, real words based on phonological, orthographic, morphological, etymological, and combined spelling skills also yielded significant differences in performance between children with and without dyslexia and may 
prove helpful when assessing spelling performance in Dutch-speaking children. Furthermore, our findings suggest use of real words (which resulted in a medium effect size for the difference between children with and without dyslexia) may be more advantageous than the use of pseudowords (which resulted in a small effect size for the difference between groups). Nevertheless, all children with dyslexia in this sample (even the oldest ones in grade 6) demonstrated below average performance on pseudoword spelling. Thus, pseudowords still might be helpful in distinguishing children with dyslexia from children without learning problems especially in older age groups. Finally, we found that being good at spelling one kind of word or pseudoword was significantly related to being good at spelling any other kind of word, especially for children with dyslexia.

\section{Limitations}

This study certainly has some limitations. First, four specific spelling skills (namely phonological, orthographic, morphological, and etymological) were studied by using words that privileged these sources of spelling knowledge to be successful in spelling them. Different classification systems for spelling errors are available, such as the taxonomy of Catach (described in Hoeflin \& Franck, 2005), the taxonomy of Masterson and Apel (2010), the model proposed by Daigle et al. (2016), the classification system used by Duranovic (2017), and the taxonomy of Rello, Baeza-Yates, and Llisterri (2017). These taxonomies inspired our approach to classify spelling words and, in line with Tops (2014), we wanted to make an even more detailed analysis of difficulties children encounter when spelling in Dutch. Thus, our results are not directly comparable to those reported by other researchers using different taxonomies (and we did not examine spelling errors per se, but rather performance on words that differed in prominent spelling skills thought required for spelling success). In addition, omissions, additions, transpositions, inversions, or substitutions of letters were considered in this study as problems with phonological skills or as phonological 
errors, in line with how Daigle et al. (2016) and Duranovic (2017) handled such errors, but in contrast with Masterson and Apel (2010), who classified omissions and additions as problems with phonological awareness.

Second, while we acknowledge that even complex words rely on some knowledge of simple mapping between letters and sounds, in this study we selected words to investigate orthographic knowledge that could not be spelled correctly based on phoneme-grapheme correspondences alone. Rather, we selected words that with complex grapheme-phoneme correspondences that could not be written based on phonological skills (e.g., 'snoei' [prune] and 'enkeling' [individual]) to investigate difficulties with orthographic skills. Moreover, Masterson and Apel (2010) noted semantic and orthographic knowledge is important for the correct spelling of homophones. In the Dutch language, the correct spelling of some homophones is in fact semantically and orthographically based, e.g., the spelling of real words with the phoneme-grapheme correspondences ei-ij/au-ou. These words share their pronunciations but have two different meanings and two different letter patterns. Although some researchers would refer to these words as simply recruiting orthographic knowledge, for the understanding of the spelling problems of children with dyslexia in Dutch, and thus for clinical practice in relatively transparent languages, we believed it would be interesting to differentiate them as a separate category from other words that recruit orthographic knowledge (Van Vreckem, 2018). Due to the necessity of access to their representations in the long-term memory, we considered these words to be based on etymological skills, as well as other non-homophonic words such as loanwords that also require storage in and retrieval from long-term memory for correct spelling.

Third, the use of a single measure of spelling and the choice of words raises caution. A replication study with other words (such as verbs) controlled for differences between subjects and words may be interesting. For instance, the spelling of verbs and of whole sentences was 
not assessed in our study, pointing to the need for future research on how knowledge of grammatical rules (e.g., inflected verb endings) and spelling must be integrated to spell verbs and sentences. In addition i might also that the knowing of the meaning of a words or the vocabulary knowledge affected the spelling ability. Those aspects were not assessed. In addition from a more conceptual perspective, it might be interesting to compare classification taxonomies and use of different word types. Moreover, it seems necessary to work toward a consensus on constructs and their definitions as well as a classification system for spelling errors and spelling words to compare studies within and across languages. We hope with our study to have added to a more detailed analysis and understanding of spelling subskills involved in successful and less successful spelling. Finally, in this study, the small sample size of children with dyslexia, with fewer girls than boys, presents a limitation, although there are fewer girls than boys with dyslexia in the population as well (Grigorenko, 2001). Additional research with more children is needed to confirm our results.

\section{CONCLUSION}

This study addressed the role of spelling skills in Dutch-speaking children with and without dyslexia in Flanders (the Dutch-speaking part of Belgium). There was a medium effect size for differences between the two groups on morphological, phonological, combined, and etymological spelling skills. The overall spelling accuracy of real words differentiated groups better than the spelling accuracy of pseudowords, but older children remained to not score above the mean on pseudowords in grade 6. Children without dyslexia performed above the mean for the whole sample on phonological, orthographic, morphological, and etymological skills by grade 4, whereas their peers with dyslexia succeeded only two years later to spell above the sample mean on most words. Children with dyslexia continued to have problems with phonological spelling skills for monosyllabic real words although the high 
exposure to these words and with pseudowords where there was no o-occurrence of form and meaning.

Our findings have practical implications for the assessment of children with dyslexia in a relatively transparent language. Although the correlations in kids with dyslexia between the subskills are pretty high, the use of pseudowords might be especially helpful in, since older children these children still seem to score below the mean of these kind of words in grade 6 . In younger children, real words that supposedly recruit morphological, phonological, combined, and etymological spelling knowledge and skills should be used to obtain a reliable identification of spelling problems. Clinicians are encouraged to be aware of the importance of the choice of spelling items in an assessment battery of dyslexia. They should not neglect the varying importance of subskills even in relatively transparent orthographies. Moreover, due to dissimilar classification systems and constructs, they should be aware that findings of spelling studies remain difficult to compare. 


\section{REFERENCES}

Angelelli, P., Judica, A., Spinelli, D., Zoccolotti, P., \& Luzzatti, C. (2004). Characteristics of writing disorders in Italian dyslexic children. Cognitive and Behavioral Neurology, 17, $18-31$.

Arfé, B., De Bernardi, B., Pasini, M., \& Poeta, F. (2012). Toward a Redefinition of Spelling in Shallow Orthographies. Phonological, Lexical, and Grammatical Skills in Learning to Spell Italian. In V.W. Berninger (Ed.), Past, present, and future contributions of cognitive writing research to cognitive psychology (pp. 359-387). New York: Psychology Press/Taylor Francis Group.

Bergmann, J., \& Wimmer, H. (2008). A dual-route perspective on poor reading in a regular orthography: Evidence from phonological and orthographic lexical decisions. Cognitive Neuropsychology, 25, 653-676.

Berninger, W.V., Nielsen, H.K., Abbott, D.R., Wijsman, E., \& Raskind, W. (2008). Writing problems in developmental dyslexia: under-recognized and under-treated. National Institutes of Health, 46, 1-21.

Bourassa, D.C., \& Treiman, R. (2014). Spelling Development and Disability in Englisch. In C.A. Stone, E.R. Siliman, B.J. Ehren, \& G.P. Wallach, G.P. (Eds.), Handbook of language and literacy. Development and disorders. (pp. 569-583). New York: The Guilford Press.

Brus, B. T., \& Voeten, M. J. M. (1999). Eén-Minuut-Test: vorm A en B [One Minute Reading Test]. Nijmegen, The Netherlands: Berkhout.

Callens, M., Tops, W., \& Brysbaert, M. (2012). Cognitive Profile of Students Who Enter Higher Education with an Indication of Dyslexia. Plos One, 7. doi: 10.1371/journal.pone.0038081 
Campbell, R. (1985). When children write nonwords to dictation. Journal of Experimental Child Psychology, 40, 133-151.

Caravolas, M., \& Voli’n, J. (2001). Phonological spelling errors among dyslexic children learning a transparent orthography: The case of Czech. Dyslexia, 7, 229-245.

Coch, D., Mytra, P., \& George, E. (2012). Behavioral and ERP evidence of word and pseudoword superiority effects in 7- and 11-year olds. Brain Research, 1486C, 68-81.

Daigle, D., Costerg, A., Plisson, A., Ruberto, N., \& Varin, J. (2016). Spelling errors in French-speaking children with dyslexia: Phonology may not provide the best evidence. Dyslexia, 22, 137-157.

De Weerdt, F., Desoete, A., \& Roeyers, H. (2013). Working memory in children with reading and/or mathematical disabilities Journal of Learning Disabilities, 46, 461-472.

Duranonvic, M. (2017). Spelling errors of dyslexic childdren in Bosnia language with transparant orthography. Journal of Learning Disabilities, 50, 591-601.

Geelhoed, J., \& Reitsma, P. (2000). PI-dictee [PI-dictation]. Lisse, The Netherlands: Harcourt Test Publishers.

Ghesquière, P. (2014). “Actualisering van het standpunt in verband met de praktijk van attestering voor kinderen met een leerstoornis in het gewoon onderwijs.," in Zorg dragen voor kinderen en jongeren met leerproblemen. Handvaten voor goede praktijk. (Leuven: Acco), 11-19.

http://www.prodiagnostiek.be/sites/default/files/L_B_EMT.pdf http://www.prodiagnostiek.be/sites/default/files/L_B_De\%20Klepel.pdf Hilte, M., \& Reitsma, P. (2006). Spelling pronunciation and visual preview both facilitate learning to spell irregular words. Annals of Dyslexia, 56, 301-318. 
Hilte, M., \& Reitsma, P. (2011). Activating the meaning of a word facilitates the integration of orthography: evidence from spelling exercises in beginning spellers. Journal of Research in Reading, 34, 333-345.

Hoeflin, G., \& Franck, J. (2005). Development of spelling skills in children with and without learning disabilities. Educational Studies in Language and Literature, 5, 175-192.

Jap, B.A.J., Borleffs, E., \& Maassen, B.A.M. (2017). Towards identifying dyslexia in Standard Indonesian: the development of a reading assessment battery. Read and Writing: An Interdiscplinary Journal, 3, 1729-1751.

Jongejan, W., Verhoeven, L., \& Siegel, L. (2007). Predictors of Reading and Spelling Abilities in First and Second Language Learners. Journal of Educational Psychology, 99, 835-851.

Joshi, R.M., \& Carreker,S. (2009). Spelling. Development, assessment and instruction. In G. Reid (Ed.), The Routledge companion to dyslexia. London: Routledge.

Kahmi, A.G., \& Hinton, L.N. (2000). Explaining individual differences in spelling ability. Topics in Language Disorders, 20, 37-49.

Keuning, J., \& Verhoeven, L. (2007). Screening for word-reading and spelling problems in elementary school: An item response theory perspective. Education and Child Psychology, 24, 44-58.

Koh, P.W., Shakory, S., Chen, X., \& Deacon, H. (2017). Morphology and spelling in French: A comparison of at-risk readers and typically developing children. Dyslexia, 23, 406427.

Kort, W., Schittekatte, M., Bosmans, M., Compaan, E. L., Dekker, P. H., \& Vermeir, G. (2005). WISC-III-NL Wechsler Intelligence Scale For Children, Derde Editie NL. Handleiding en Verantwoording. Amsterdam: Harcourt Test Publishers/Nederlands Instituut voor Psychologen. 
Landerl, K., \& Wimmer, H. (2000). Deficits in phoneme segmentation are not the core problem of dyslexia: Evidence from German and English children. Applied Psycholinguistics, 21, 243-262.

Masterson, J. J., \& Apel, K. (2000). Spelling assessment: Charting a path to optimal intervention. Topics in Language Disorders, 20, 50-65.

Masterson, J. J., \& Apel, K. (2010). Linking characteristics discovered in spelling assessment to intervention goals and methods. Learning Disability Quarterly, 33,185-198.

Mather, N., \& Wending, B. J. (2012). Essentials of dyslexia assessment and intervention. New Jersey: John Wiley \& Sons, Inc.

Nikolopoulos, D., Goulandris, N., \& Snowling, M. (2003). Developmental dyslexia in Greek. In N. Goulandris (Ed.), Dyslexia in different languages (pp. 53-67). London: Whurr.

Reitsma, P. (1983). Printed word learning in beginning readers. Journal of Experimental Child Psychology, 36, 321-339.

Rello, L., \& Beaza-Yates, R., \& Llisterri, J. (2017). A resource of errors written in Spanish by people with dyslexia and its linguistic, phonetic and visual analysis. Language Resources \& Evaluation, 51, 379-408.

Rispens, J.E., McBride-Chang, C., \& Reitsma, P. (2008). Morphological awareness and early and advanced word recognition and spelling in Dutch. Reading and Writing: An Interdisciplinary Journal, 21, 587-607.

Tellings, A., \& Bouts, L. (2011).Dutch elementary school children's attribution of meaning to written pseudowords. Reading and Writing: An Interdisciplinary Journal, 24, 801-812

Tolchinsky, L. Liberman, G., \& Alonso-Cortés Fradejas, M. (2015). Explaining first graders’ achievements in spelling and word separation in shallow orthographies. Journal of Writing Research, 6, 270-316. 
Tops, W., Callens, M., Bijn, E., \& Brysbaert, M. (2014). Spelling in adolescence with dyslexia: Errors and modes of assessment. Journal of Learning Disabilities, 47, 295306.

Van den Bos, K. P., Spelberg, L. H. C., Scheepstra, A. J. M., \& de Vries, J. R. (1994). De Klepel: vorm A en B. Nijmegen, The Netherlands: Berkhout.

Van Steenbrugge, H., Valcke, M., \& Desoete, A. (2010). Mathematics learning difficulties in primary education: teachers' professional knowledge and the use of commercially available learning packages. Educational Studies, 36, 59-71.

Van Vreckem, C. (2018). Spelling skills in pseudowords and existing words in Dutch speaking children with and without dyslexia. Paper in the symposium 'Spelling in different languages: Do we speak and write the same language? $42^{\text {nd }}$ Annual International Association of Research of Learning Disabilities (IARLD) conference for Reseach in Learning Disabitities. 3th of July 2018 Gent.

Van Vreckem, C., \& Desoete, A. (2016). Spelling pseudo and existing words in children with dyslexia in a semi-transparent. Paper in the symposium 'Spelling across languages'. Austin: IARLD-conference: 28.06.2016-30.06.2016

Van Vreckem, C., \& Desoete, A. (2016). ST 1-6. Spellingtest voor kinderen van het eerste tot het zesde leerjaar (Spelling test for children from the 1st until the 6th grade). Gent: Lannoo, Academia Press.

Wechsler, D. (1991). Wechsler intelligence scale for children (3rd ed.). New York: Psychological Corporation.

Wimmer, H., \& Landerl, K. (1997). How learning to spell German differs from learning to spell English. In C.A. Perfetti, L. Rieben, \& M. Fayol (Eds.), Learning to spell: Research, theory, and practice across languages (pp. 81-96). Mahwah, NJ: Lawrence Erlbaum. 
Ziegler, J.C., \& Goswami, U. (2005). Reading acquisition, developmental dyslexia, and skilled reading across languages: A psycholinguistic grain size theory. Psychological Bulletin, 13(1), 3-29. 
亨

\begin{tabular}{|c|c|c|c|c|}
\hline 会 & 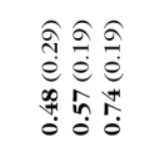 & $\begin{array}{l}\hat{r} \\
\hat{e} \\
o \\
o \\
0 \\
0\end{array}$ & 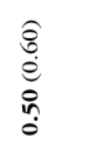 & 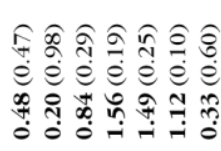 \\
\hline 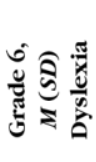 & 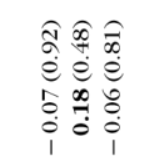 & $\begin{array}{l}E \\
E \\
E \\
0 \\
0 \\
i \\
1\end{array}$ & $\begin{array}{l}\widehat{a} \\
\stackrel{8}{0} \\
\stackrel{+}{0} \\
\dot{0}\end{array}$ & 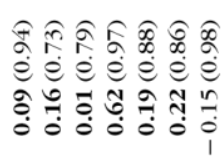 \\
\hline$\hat{\mathrm{z}}$ & 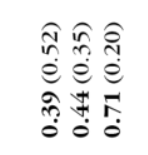 & 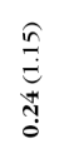 & $\begin{array}{l}\hat{0} \\
\stackrel{0}{0} \\
\stackrel{0}{0} \\
0\end{array}$ & 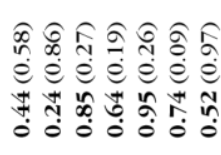 \\
\hline 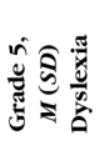 & 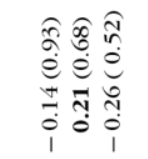 & $\begin{array}{c}\frac{r}{\infty} \\
e \\
0 \\
0 \\
0 \\
i \\
1\end{array}$ & $\begin{array}{l}\text { E } \\
\stackrel{0}{0} \\
\stackrel{a}{0}\end{array}$ & 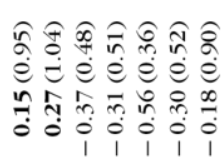 \\
\hline 会 & 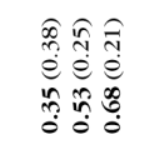 & $\begin{array}{l}\widehat{\infty} \\
\stackrel{\infty}{e} \\
\stackrel{0}{0}\end{array}$ & 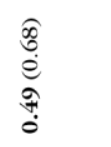 & 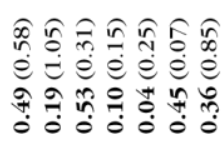 \\
\hline 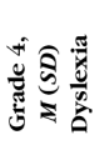 & 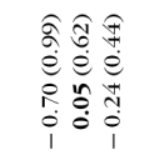 & $\begin{array}{c}\hat{f} \\
0 \\
0 \\
0 \\
i \\
1\end{array}$ & $\begin{array}{c}6 \\
\infty \\
\stackrel{0}{0} \\
0 \\
0 \\
0 \\
1\end{array}$ & 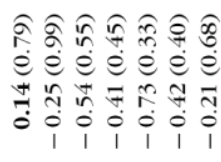 \\
\hline 会 & 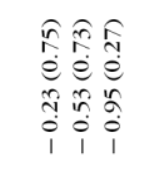 & 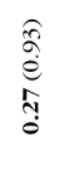 & $\begin{array}{l}\hat{f} \\
\stackrel{0}{0} \\
0 \\
i \\
i\end{array}$ & 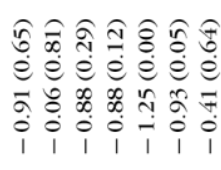 \\
\hline 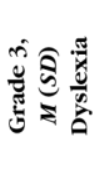 & 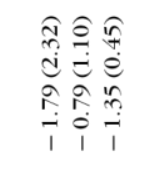 & $\begin{array}{l}\sqrt{\hat{T}} \\
\stackrel{0}{0} \\
0 \\
0 \\
0 \\
1\end{array}$ & 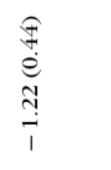 & 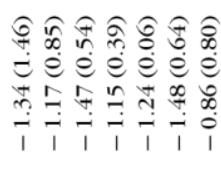 \\
\hline 令 & 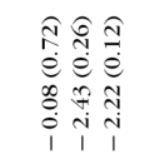 & $\begin{array}{c}\text { f } \\
e \\
e \\
\infty \\
\infty \\
0 \\
i\end{array}$ & 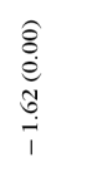 & 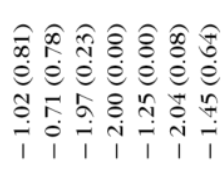 \\
\hline 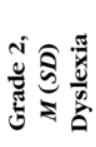 & 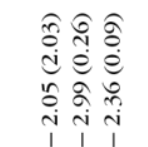 & $\underset{\substack{0 \\
\stackrel{0}{2}}}{\stackrel{0}{+}}$ & $\begin{array}{l}\widehat{\delta} \\
\stackrel{e}{0} \\
\substack{1 \\
-1}\end{array}$ & 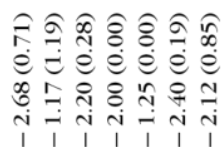 \\
\hline & \multicolumn{4}{|c|}{ 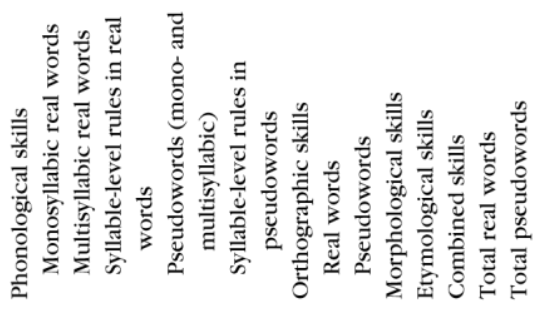 } \\
\hline
\end{tabular}

